J. Phys. IV France 127 (2005) 145-149

(C) EDP Sciences, Les Ulis

DOI: $10.1051 /$ jp4:2005127022

\title{
Enlèvement de particules par laser impulsionnel
}

\author{
D. Grojo, P. Delaporte et A. Cros
}

\author{
LP3, UMR 6182 CNRS, Université de la Méditerranée, Case 917, \\ 13288 Marseille Cedex 9, France
}

\begin{abstract}
Résumé. L'enlèvement de particules par laser impulsionnel (ns) est étudié expérimentalement. Des efficacités supérieures à $90 \%$ sont obtenues pour l'enlèvement de particules de polystyrene (PS) de diamètre $385 \mathrm{~nm}$ sur des surfaces de $\mathrm{Si}$ et $\mathrm{Cu}$. Les fluences seuils d'enlèvement sont déterminées et comparées à une modélisation de l'expansion thermique du substrat. L'interprétation est discutée en terme de mécanismes fondamentaux envisageables responsables de l'éjection des particules.
\end{abstract}

\section{INTRODUCTION}

Le nettoyage de substrats revêt un enjeux important dans de nombreux domaines comme l'optique, l'optoélectronique et la décontamination nucléaire [1] ou bactériologique. Il va jusqu'à être une priorité stratégique pour les fabricants de microélectronique. En effet, ce sont les procédés de fabrication euxmêmes (gravure, polissage mécano-chimiques, ...) qui sont responsables de la majeure partie de la contamination [2] et qui affectent ainsi directement la performance, la fiabilité des composants et les rendements de production. Avec l'augmentation continue de la densité d'intégration des circuits intégrés, la taille critique de particule polluante acceptable ne cesse de baisser. Comme le montre la table 1, l'ITRS Roadmap* préconise déjà la nécessité d'enlever des particules de tailles inférieures à $50 \mathrm{~nm}$.

Dans ce contexte, l'utilisation de lasers impulsionnels (ns) se révèle une alternative prometteuse aux procédés mécano-chimiques actuels dans la mesure où cette méthode est efficace et peu polluante. De plus, ce procédé, basé sur l'expansion thermique des particules et/ou du substrat doit être en mesure de ne pas affecter la surface à nettoyer. Cette étude consiste donc à améliorer la compréhension du processus d'enlèvement de particules par laser de manière à l'optimiser en vue de futures applications industrielles.

Tableau 1. Exigences des performances de nettoyage d'après l'ITRS Roadmap, édition de 2001.

\begin{tabular}{|l|c|c|c|c|}
\hline Front-end; Face avant wafer & & & & \\
\hline Année & $\mathbf{2 0 0 4}$ & $\mathbf{2 0 0 6}$ & $\mathbf{2 0 1 0}$ & $\mathbf{2 0 1 8}$ \\
\hline DRAM $1 / 2$ Pitch & 90 & 70 & 45 & 18 \\
\hline Diamètre wafer (mm) & 300 & 300 & 300 & 450 \\
\hline Taille critique de particules (nm) & 45 & 35 & 22,5 & 9 \\
\hline Particules (cm ${ }^{-2}$ ) & 0,0217 & 0,0185 & 0,025 & 0,0215 \\
\hline Particules (\#/wafer) & 75 & 64 & 86 & 168 \\
\hline
\end{tabular}

\section{DISPOSITIF EXPÉRIMENTAL}

Le dispositif expérimental est basé sur une source laser excimère (EXI CILAS UV635) à $308 \mathrm{~nm}$ ayant une durée d'impulsion $\tau=60 \mathrm{~ns}$ et une énergie supérieure à $1 \mathrm{~J}$. Les expériences sont menées avec un taux de répétition $v=1 \mathrm{~Hz}$. L'énergie est ajustée par l'utilisation de miroirs extracteurs calibrés à

\footnotetext{
* L'organisme International Technology Roadmap for semiconductors prédit les exigences de l'industrie des semiconducteurs pour les 15 années à venir. Il est composé de la Semiconductor Industry association (SIA), la European Electronic Component Association (EECA) et leurs homologues japonaise, coréenne et taîwanaise.
} 
308 nm (rôle d'atténuateurs). Un masque rectangulaire est imagé sur la surface à l'aide d'une lentille $(f=600 \mathrm{~mm})$ de manière à obtenir une zone irradiée de taille $5.4 \times 5.8 \mathrm{~mm}^{2}$. Grâce au profil quasitop-hat du faisceau, la densité d'énergie $F_{l a s}$ est considérée homogène sur la surface d'irradiation. La cible est perpendiculaire au faisceau incident.

Les substrats utilisés sont des cibles $10 \times 10 \mathrm{~mm}^{2}$ polies de $\mathrm{Si}, \mathrm{Cu}$ et Al. Les particules utilisées pour cette étude sont des microsphères de polystyrene (PS) calibrées de diamètres $385 \mathrm{~nm}$ (G-KISKER). L'enduction des particules est réalisée à l'aide d'un « spin-coater » (LAURELL WS-400A-8NPP) après raréfaction des microsphères dans le propanol-2 (IPA). Ce solvant offre un fort taux d'évaporation et un effet mouillant important permettant une distribution quasi-homogène des paticules sur le substrat. Avec une vitesse de rotation constante de $4000 \mathrm{rpm}$, on obtient des densités de particules moyenne de l'ordre de $10^{6}$ particules $/ \mathrm{cm}^{2}$ avec une proportion de particules isolées supérieure à $90 \%$. Les irradiations comportent chacune 10 tirs pour des fluences variant de 0.15 à $3 \mathrm{~J} \mathrm{~cm}^{-2}$. La détermination de l'efficacité d'enlèvement $\eta$ est réalisée par microscopie optique (ZEISS Axiotech, objectif Epiplan $\times 100 / 0.75)$ selon la relation :

$$
\eta=1-\frac{N}{N_{0}}
$$

où $\mathrm{N}_{0}$ est le nombre de particules avant irradiation et $\mathrm{N}$ le nombre de particules restantes après irradiation (figure 1). Pour limiter l'incertitude et obtenir des résultats statistiquement significatifs, plusieurs images sont enregistrées avant et après l'irradiation. Ainsi, les estimations sont toujours réalisées sur plus d'un millier de particules.

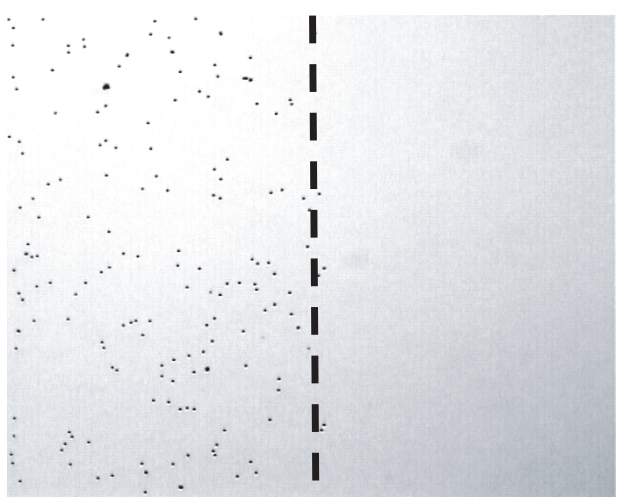

Figure 1. Particules de PSL sur surface de Si avant et aprés irradiation pour Flas $\cong 650 \mathrm{~mJ} \mathrm{~cm}^{-2}$.

\section{RÉSULTATS}

La figure 2 présente l'efficacité d'enlèvement de particules de PS sur substrat de Si et Cu en fonction de la fluence laser appliquée. On note des comportements similaires avec une fluence seuil plus basse pour le silicium. Pour une fluence de $0.5 \mathrm{~J} \mathrm{~cm}^{-2}$, l'efficacité d'enlèvement excède $90 \%$ pour le silicium alors qu'elle est toujours quasi-nulle pour le cuivre. La force dominant l'adhésion des particules de taille sub-micronique sur substrat sec est la force de Van der Waals donnée par [3] :

$$
F_{\text {Adh. }}=\frac{H a}{6 z_{0}^{2}}\left(1+\frac{r_{c}^{2}}{a z_{0}}\right)
$$

où $H$ est la constante de Hamaker entre les matériaux 1 (substrat) et 2 (particule) donnée par $H=\left(H_{1} \times H_{2}\right)^{1 / 2}, a$ le rayon de la particule, $r_{c}$ le rayon de la zone de contact et $z_{0}$ la distance entre la surface et la particule $(\approx 0.4 \mathrm{~nm})$. Les expériences étant toutes menées avec des particules de PS le terme de déformation de la particule est considéré quasi-constant. La valeur de la constante de Hamaker pour les métaux étant de $\approx 4 \times 10^{-19} \mathrm{~J}$ [4] alors qu'elle est de $2.5 \times 10^{-19} \mathrm{~J}$ [5] pour le silicium, on 


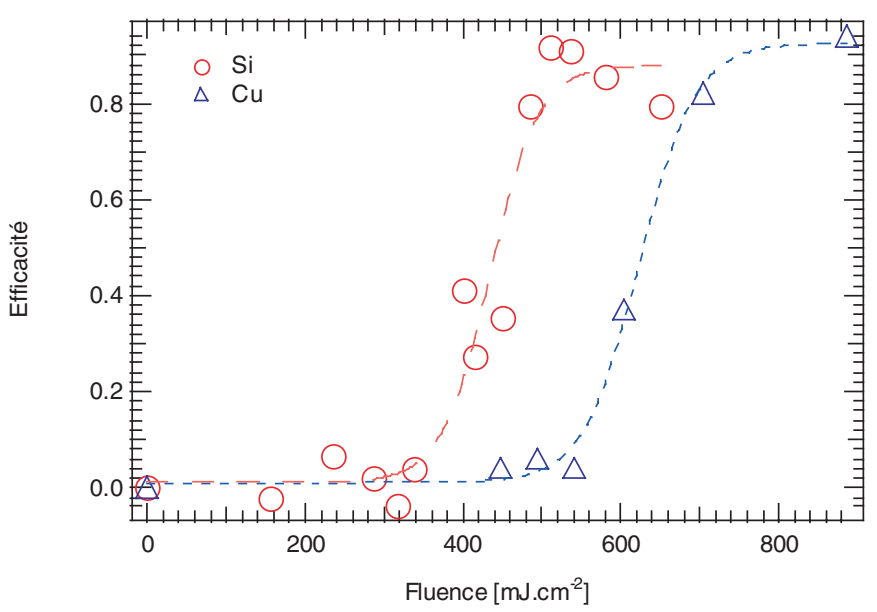

Figure 2. Efficacité d'enleèvement en fonction e la fluence laser appliquée pour 10 tirs sur Si et Cu.

prévoit donc une adhésion peu différente pour les différents matériaux. On prévoit en effet une adhésion des particules environ 1.3 fois plus importante pour $\mathrm{Cu}$ et $\mathrm{Al}$ que pour $\mathrm{Si}$. Le seuil d'enlèvement étant $\approx 100 \mathrm{~mJ}$ plus faible pour $\mathrm{Si}$ que pour $\mathrm{Cu}$, la force d'enlèvement pour une fluence donnée s'exerçant sur les particules est significativement plus importante lorsque elles sont déposées sur un substrat de Si.

Comme le montre la table 2, pour l'aluminium, l'efficacité n'excède $50 \%$ qu'à partir de fluences supérieures à $2.5 \mathrm{~J} \mathrm{~cm}^{-2}$. Aucun endommagement n'a été constaté pour des fluences allant jusqu’à $3 \mathrm{~J} \mathrm{~cm}^{-2}$ pour ce même matériau.

Tableau 2. Fluences correspondant à 50\% d'enlèvement pour $\mathrm{Si}, \mathrm{Cu}$ et $\mathrm{Al}$.

\begin{tabular}{|c|c|c|c|}
\hline & Si & Cu & Al \\
\hline$F_{\eta=50 \%}\left(\mathrm{~J} \mathrm{~cm}^{-2}\right)$ & 0,44 & 0,54 & $\approx 2,5$ \\
\hline
\end{tabular}

\section{EXPANSION THERMIQUE DU SUBSTRAT}

L'hypothèse du mécanisme responsable de l'enlèvement la plus souvent rencontrée est l'expansion thermique du substrat et/ou des particules [6]. La longueur de pénétration optique du PS étant de $10-10^{2} \mu \mathrm{m}$, c'est à dire largement plus grande que la taille de la particule, il n'y a pas d'absorption significative par la particule [7] et on peut ne prendre en compte que l'expansion du substrat. L'évolution de la position de la surface par rapport à sa position initiale est donnée par :

$$
l(t)=\int_{0}^{\infty} \beta(T) T(z, t) d z
$$

où $\beta(T)$ est le coefficient d'expansion thermique unilatéral. La conservation de l'energie nous permet alors d'écrire:

$$
A \int_{0}^{t} I\left(t^{\prime}\right) d t^{\prime}=\rho \int_{0}^{\infty} C_{P}(T) T(z, t) d z
$$

avec $A=1-R$ l'absorptivité de la surface, $I$ l'intensité lumineuse, $\rho$ la masse volumique et $C_{T}$ la chaleur spécifique du matériau. Supposant alors que la loi de Grüneisen donnée par $\beta / C_{T}=$ const. est vérifiée ( $\pm 10 \%$ pour le silicium [6]), en combinant (3) et (4), on obtient les expressions du déplacement, de la 
vitesse et de l'accélération de la surface:

$$
\begin{aligned}
& l(t)=\frac{\beta A}{C_{T} \rho} \int_{0}^{t} I(t) d t \\
& v(t)=\frac{\beta A}{C_{T} \rho} I(t) \\
& a(t)=\frac{\beta A}{C_{T} \rho} \frac{d I}{d t}(t)
\end{aligned}
$$

La figure 3 donne le déplacement, la vitesse et l'acceleration d'une surface de silicium pour une impulsion laser "théorique" de durée $30 \mathrm{~ns}\left(\lambda_{\text {las }}=308 \mathrm{~nm}\right)$ avec une fluence laser de $0.5 \mathrm{~J} \mathrm{~cm}^{-2}$. Ce modèle a été validé en comparant les résultats à la résolution numérique de l'équation de la chaleur puis le calcul de (3).

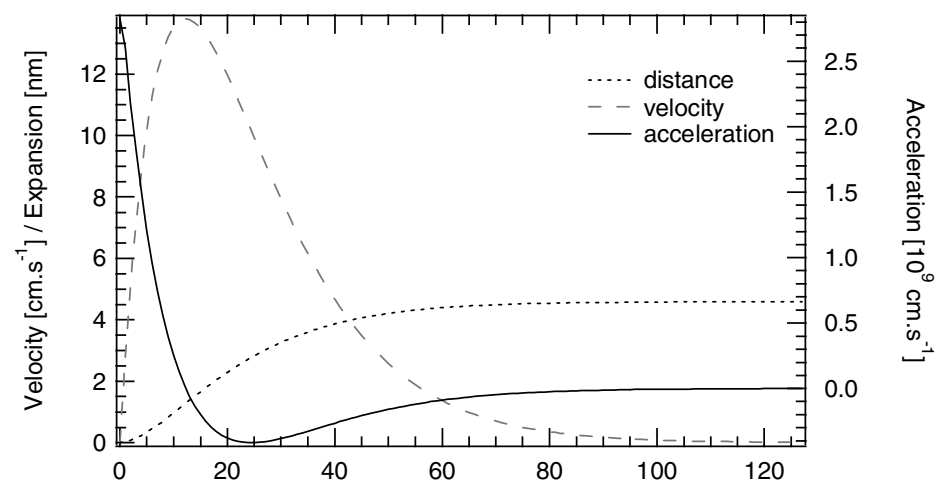

Figure 3. Accélération vitesse et déplacement d'une surface silicium pour Flas $=0.5 \mathrm{~J} \mathrm{~cm}^{-2}$.

La force s'exerçant sur la particule est donnée par la somme de la force d'adhésion (négative) et de la force d'inertie donnée par $-m . a(t)$ où $m$ est la masse de la particule. Dans le cas d'un enlèvement de particule dans le régime de la force d'inertie, on a alors décollement lorsque la force s'exerçant sur cette particule devient positive c'est à dire lorsque :

$$
a(t)<-\frac{\left|F_{A D H}\right|}{m}
$$

C'est donc lors de la décélération de la surface que peut se produire l'éjection de la particule dans ce régime. Au regard de ce modèle, le temps de « descente » de l'impulsion est donc un paramètre capital. La figure 4 montre l'accélération d'un substrat de $\mathrm{Si}, \mathrm{Cu}$ et $\mathrm{Al}$ pour une fluence laser de $0.5 \mathrm{~J} . \mathrm{cm}^{-2}$. L'aluminium et le cuivre ayant des coefficients de dilatation thermique beaucoup plus important que le silicium, ces matériaux devraient se révéler de meilleurs candidats pour le nettoyage à sec. Comme le montre la figure 4, c'est le cas pour le cuivre mais la faible absorptivité de l'aluminium $(<0.1)$ fait qu'il se comporte de façon similaire au silicium pour une fluence donnée.

La force d'inertie subie par la particule est environ 3.5 fois plus importante pour un substrat de $\mathrm{Cu}$ que pour $\mathrm{Si}$ et $\mathrm{Al}$. Au regard de ce modèle, contrairement aux constatations expérimentales, le seuil d'enlèvement le plus faible devrait donc être observé pour un substrat de $\mathrm{Cu}$. De plus, L'écart relativement important entre les comportements expérimentaux de Si et Al ne peut pas être décrit par ce modèle.

\section{RÉSUMÉ ET CONCLUSION}

Les seuils d'enlèvement expérimentaux de particules de PS $(\varnothing=385 \mathrm{~nm})$ suivent $F_{S}{ }^{S i}<F_{S}{ }^{C u} \ll F_{S}{ }^{A l}$. La force d'inertie s'exerçant sur la particule résultant de l'expansion thermique du substrat a été estimée 


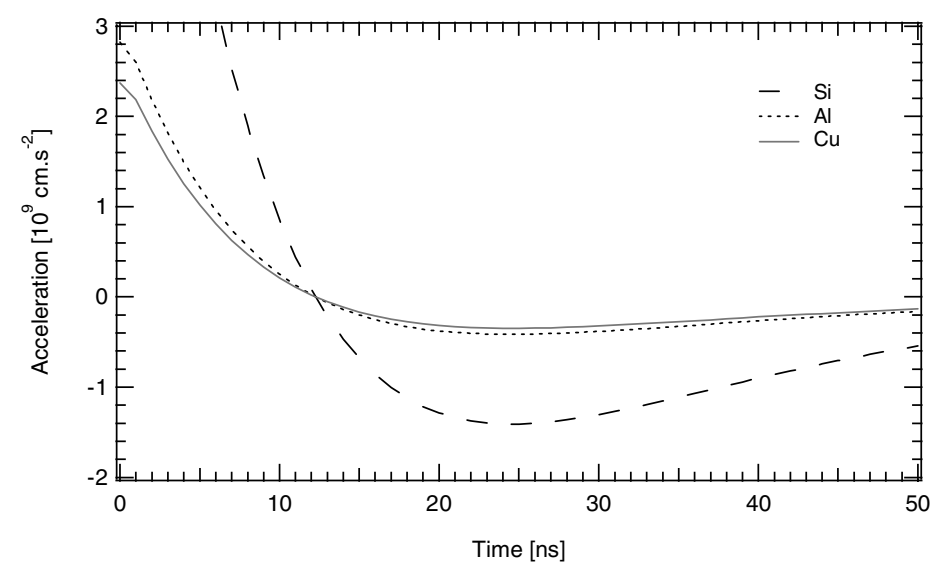

Figure 4. Accélération d'une surface de $\mathrm{Si}, \mathrm{Cu}$ et $\mathrm{Al}$ pour Flas $=0.5 \mathrm{~J} \mathrm{~cm}^{-2}$.

$\approx 3.5$ fois plus importante pour $\mathrm{Cu}$ que pour $\mathrm{Si}$ ou $\mathrm{Al}$ alors que les forces d'adhésion pour chacun des matériaux sont considérées ici comme similaires. Au regard de ces résultats, il n'est pas possible de valider l'hypothèse selon laquelle l'expansion thermique du substrat est le mécanisme principal responsable de l'enlèvement des particules.

On remarque de plus une certaine corrélation entre les seuils d'endommagement des matériaux et les seuils d'enlèvement des particules. Mosbacher a suggéré que l'éjection des particules pouvait être due à l'ablation locale du substrat [8]. Ce phénomène peut se produire pour des fluences inférieures à son seuil d'endommagement à cause de l'effet d'autofocalisation des particules décrit par la théorie de Mie [9]. Cependant dans notre étude, aucune présence de cratère n'a été constatée par microscopie électronique sur la surface de Si après irradiation laser.

Il est encore prématuré d'exclure l'hypothèse d'un enlèvement résultant de l'expansion thermique des matériaux dans la mesure où des paramètres tels que la rugosité, l'hydrométrie, l'oxydation du substrat ne sont pas parfaitement maîtrisés et qu'ils peuvent modifier fortement la valeur des forces d'adhésion voire changer leurs natures (liaisons $\mathrm{H}, \ldots$... Des expériences complémentaires vont être menées pour étudier l'influence de paramètres tels que la forme (temps de «descente »), la durée d'impulsion laser, l'angle d'incidence du faisceau, la longueur d'onde d'irradiation, etc... Elles devraient nous permettre de déterminer les principaux mécanismes responsables de l'éjection des particules, et ainsi d'optimiser le procédé de dépoussiérage par laser.

\section{Bibliographie}

[1] P. Delaporte, M. Gastaud, W. Marine, M. Sentis, O. Uteza, P. Thouvenot, J.L. Alcaraz, J.M. Le Samedy, D. Blin, Appl. Surf. Sci. 298 (2003) 208

[2] A. Baudrant, F. Tardif, C. Wyon, Caractérisation et nettoyage du silicium (Hermes Science Publications, Paris, 2003) pp. 19-27

[3] R.A. Bowling, J. Electrochem. Soc. 132 (1985) 2209

[4] C. Curran, J.M. Lee, K.G. Watkins, Optics and Lasers in Engineering (2002)

[5] Y.W. Zheng, B.S. Luk'yanchuk, Y.F. Lu, W.D. Song, Z.H. Mai, J. Appl. Phys. 90 (2001)

[6] N. Arnold, Appl. Surf. Sci. 15 (2003) 208

[7] M. Meunier, X. Wu, F. Beaudoin, E. Sacher, M. Simard-Normandin, SPIE 3618 (1999)

[8] M. Mosbacher, H.J. Münzer, J. Zimmermann, J.Solis, J. Boneberg, P. Leiderer, Appl. Phys. A 72 (2001) 41

[9] M. Born, E. Wolf, Principle of optics 7th (expanded) edition (Cambridge, 1999) pp.759-789 